\section{Flavio Dalla Vecchia}

Università Cattolica del Sacro Cuore di Milano

flavio.dallavecchia@fastwebnet.it

DOI: http://dx.doi.org/10.12775/BPTh.2015.027

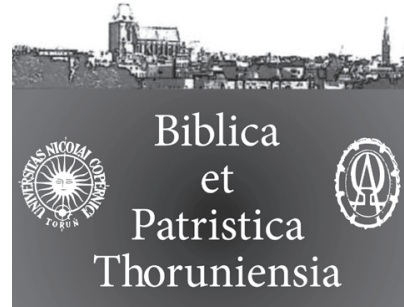

8 (2015) 4: 87-97

ISSN (print) 1689-5150

ISSN (online) 2450-7059

\title{
Giosuè, la guida che medita e mette in pratica la Torah
}

\section{Joshua, the guide who meditates and practicing the Torah}

\section{Jozue, przewodnik, który medytuje i praktykuje Torę}

\begin{abstract}
The research on the Book of Joshua usually has not centred about the figure of main character, usually because he is predominantly styled as Moses redux, but also because the narrator never relates the substance of thoughts or reports an interior monologue by him. Recently some suggests that Joshua is styled in his Book as second servant of Yhwh (in the light of the title ascribed to him in Josh. 24:29), a prophet (according the presentation in Ch. 1), or - if one accepts the hypothesis of Deuteronomistic History - a sort of prototypical Josiah.

The contribution shows that, in light of the general outlook of the Book, the main character doesn't become clear in relation to his predecessor, neither in light of the historical implementations in the ensuing narrative, notably Josiah.

Joshua typify a model of leader who will not find real followers, tacitly preparing the reader of the subsequent history for the unlucky end of the monarchic experience. The postexilic community, which waits for the realization of the divine promises of renewal, will not be ruled by a king, but will have as permanent point of reference - for the guides and for the members - the Book, and anyone will be called to take on the task of the leader, should - like Joshua - hear, meditate and act in accordance of the Torah.
\end{abstract}

Steszczenie. Badania nad Księgą Jozuego nie skupiają się na postaci głównego bohatera, zwykle dlatego że jest ona przede wszystkim stylizowana na Mojżesza redux, ale także dlatego że nigdy narrator nie relacjonuje istoty jego myśli czy nie podaje jego wewnętrznego monologu. Ostatnio niektórzy sugerują, że Jozue jest stylizowany w księdze na drugiego sługę Yhwh (w świetle tytułu przypisanego mu w Joz 24,29), na proroka (zgodnie z prezentacją w rozdz. 1) czy - jeśli akceptuje się hipotezę historii dueteronomistycznej - na rodzaj prototypowego Jozjasza.

Artykuł pokazuje, że - w świetle ogólnej perspektywy Księgi - główny bohater nie staje się wyrazisty w relacji do swojego poprzednika, ani - w świetle historycznych wdrożeń w późniejszej narracji - w relacji do Jozjasza. 
Jozue uosabia model przywódcy, który nie znajduje prawdziwych naśladowców, milcząco przygotowuje czytelnika do późniejszej historii pechowego końca doświadczenia monarchicznego. Powygnaniowa społeczność, która czeka na realizację Bożą obietnicę odnowy, nie będzie rządzona przez króla, lecz będzie miał jako stały punkt odniesienia Księgę dla przewodników i dla członków, i każdy, który będzie wezwany do podjęcia się roli lidera, musi jak Jozue słuchać, medytować i czynić wszystko zgodnie z Torą.

Keywords: Joshua; the Book of Joshua; the guide; the leader; the Torah.

Słowa kluczowe: Jozue; Księga Jozuego; przewodnik; przywódca; Tora.

U na recente tesi sul protagonista del libro di Giosuè rileva come tale personaggio sia stato raramente oggetto d'indagine per sé, ${ }^{1}$ dato che gli studi su quest'opera si sono in genere concentrati sullo sfondo storico e sulle problematiche letterarie. Va dato merito all'autore della menzionata ricerca di non aver affrontato la tematica sulla base della ricostruzione storica del personaggio (chi era Giosuè per i suoi contemporanei?), cercando invece di delineare la sua figura alla luce del libro e dell'intreccio narrativo entro il quale è situato. In tal modo risalta l'autonomia di Giosuè rispetto a Mosè (cioè egli non è solo il successore di Mosè ${ }^{2}$ - titolo peraltro assente nel libro biblico), pure risalta, se si legge il seguito della vicenda, la netta differenza tra Giosuè e i giudici e i re che fungeranno da guide per il popolo - nonostante i suggestivi tentativi di Porter e Nelson. ${ }^{3}$

Una lettura anche solo marginale del percorso narrativo mostra infatti che, mentre all'inizio la figura del protagonista è qualificata alla luce del suo rapporto con Mosè (cf. Gs 1,1: «Dopo che Mosè, servo ('ébed) di Yhwh, morì, Yhwh disse a Giosuè, figlio di Nun, servitore ( $m^{e}$ šārēt $)$ di Mosè» $\left.{ }^{4}\right)$, alla fine del libro anche Giosuè riceve l'appellativo «servo di Yhwh ('éed)»(Gs 24,29). La designazione "servitore" ha qui la funzione di legittimare la successione di Giosuè a Mosè, come attestano casi analoghi (cf. 1Sam 3,1: Samuele ed Eli; 1Re 19,21: Eliseo ed Elia). Non così l'appellativo «servo ('ebed)» che nell'AT è attribuito a diversi personaggi, oltre a Mosè e Giosuè: Abramo (Gen 26,24), Giacobbe

1 P. Béré, Le second Serviteur de Yhwh.

2 Cf. Ch. Schäfer-Lichtenberger, Josua und Salomo. Sulla "successione", cf. inoltre: R. Vignolo, «Antitipo come controtipo. Giosuè», pp. 191-203.

3 J. Porter, «The Succession of Joshua», pp. 102-132; R.D. Nelson, «Josiah in the Book of Joshua», s. 531-540; idem, Joshua, pp. 21-22. 119.

4 Trad. mia. Quando non segnalato, seguo la traduzione CEI 2008. 
( $E z$ 28,25; 37,25), Kaleb (Nm 14,24), David (2Sam 3,18; 7,5.8; 1Re 11,13), Giobbe $(G b 1,8 ; 2,3 ; 42,7.8)$, Isaia (Is 20,3), Zorobabele (Ag 2,23), il Germoglio

$(Z c 3,8)$. E inoltre applicato in senso collettivo all'intero Israele, specialmente nella seconda parte del libro di Isaia (cf. Is 40-55), oltre che a profeti (cf. $2 \operatorname{Re} 17,13.23 ; 21,10 ; 24,2)$. Da tale rassegna emerge che l'appellativo, più che indicare una carica o un ruolo, definisce la particolare relazione del designato rispetto a Dio.

Che cosa è avvenuto al personaggio Giosuè nel percorso narrativo per ottenere tale titolo? Il personaggio è cresciuto, ha trovato una sua identità?

Se si risale all'indietro nella sequenza narrativa del Pentateuco, è evidente la dipendenza di Giosuè dal suo predecessore. In primo luogo va tenuto conto del titolo «servitore ( $\left.m^{e} \check{s} \bar{a} r \bar{r} t\right)$ di Mosè», il quale richiama circostanze in cui Giosuè è designato in tal modo (Es 24,13; 33,11; Nm 11,28), ricordando al lettore la stretta associazione di Giosuè a eventi in cui Mosè esercita la sua leadership: quando Yhwh consegna le tavole di pietra al Sinai (Es 24,12-14), nelle occasioni in cui Yhwh parla a Mosè "faccia a faccia» presso la tenda del convegno (Es 33,7-11) e quando lo spirito di Yhwh discende sugli anziani d'Israele (Nm 11,16-30).

La dipendenza da Mosè è inoltre sottolineata quando si narra l'investitura di Giosuè in Nm 27,12-23. Mosè ormai anziano, impedito di entrare nella terra promessa, chiede a Dio: «Il Signore, il Dio della vita di ogni essere vivente, metta a capo di questa comunità un uomo che li preceda nell'uscire e nel tornare, li faccia uscire e li faccia tornare, perché la comunità del Signore non sia un gregge senza pastore» ( $\mathrm{Nm}$ 27,16-17). Tale richiesta è esaudita da Dio che sceglie Giosuè come colui al quale conferire «una parte della tua autorità $(h o ̂ d)^{5}$ » $(\mathrm{Nm}$ 27,20). Infine «Mosè fece come il Signore gli aveva ordinato; prese Giosuè e lo fece comparire davanti al sacerdote Eleàzaro e davanti a tutta la comunità; pose su di lui le mani e lo insedio,${ }^{6}$ come il Signore aveva detto per mezzo di Mosè» (Nm 27,22-23).

Al termine dei suoi giorni infine, Mosè esorta il suo assistente con queste parole: «Sii forte e fatti animo, perché tu condurrai questo popolo nella terra che il Signore giurò ai loro padri di darvi: tu gliene darai il possesso. Il Signore stesso cammina davanti a te. Egli sarà con te, non ti lascerà e non ti abbando-

5 B.A. Levine, Numbers 21-36., p. 351: «Hebrew hôd is said both of God and of eartly kings and heroes. It is an aura that commands awe and respect».

6 Cf. B.A. Levine, Numbers 21-36, p. 351: «In certain of its usages, the verb șiwwāh "to command" has the sense of empowering or appointing someone to a position of authority. Thus, the God of Israel variously empowers leaders and prophets over his people (2 Sam 7:7,11; 1 Re 1:35)». Si veda anche P.J. Budd, Numbers, pp. 304-305. 
nerà. Non temere e non perderti d'animo!» (Dt 31,7-8); «Sii forte e coraggioso, poiché tu introdurrai gli Israeliti nella terra che ho giurato di dare loro, e io sarò con te» (Dt 31,23). Risalta dunque come fino a Gs 1 il rapporto Yhwh-Giosuè sia di fatto mediato da Mosè. Non così se si guarda alle prime battute del libro che porta il suo nome; qui infatti Yhwh parla direttamente a Giosuè, proprio come a Mosè, e lo rassicura che sarà con lui come è stato con Mosè $(G s 1,5)$. Vi è però chi vede riconfermata la subordinazione a Mosè proprio sulla base di questa scena, ${ }^{7}$ sia per la reiterata limitazione del titolo «servo di Yhwh» a Mosè sia perché Yhwh conferma gli ordini impartiti da Mosè e ingiunge al nuovo leader di osservarli diligentemente (Gs 1,7-8).

E qui inoltre introdotto un elemento che nel seguito della narrazione biblica avrà un notevole peso, il riferimento al seeper hattôrâ $(G s 1,8)$ che rappresenterà il criterio di valutazione della condotta dei successivi leader del popolo. È la legge proclamata da Mosè nel paese di Moab $(D t 1,3-5)$ e messa per iscritto per essere letta ogni sette anni nell'assemblea del popolo (Dt 31,9-13); il documento era conservato presso l'arca dell'alleanza (Dt 31,24-26). La richiesta che Dio formula a Giosuè ne innalza la figura a livello regale, giacché è in linea con quanto $D t 17,18-19$ prescrive per il re: «Quando siederà sul trono del suo regno, trascriverà per sé su un libro una copia di questa legge, secondo l'esemplare che è presso i sacerdoti leviti: essa sarà con lui, la leggerà tutti i giorni della sua vita, affinché impari a temere il Signore suo Dio, a osservare tutte le parole di questa legge e questi ordinamenti». Anche se Mosè è morto, la parola da lui trasmessa al popolo permane in un documento; in tal modo egli continua a guidare Israele, sebbene non di persona, ma tramite colui che fedelmente esegue il compito che gli è stato assegnato.

La cornice narrativa del libro, che insiste sulla fedeltà di Giosuè (cc. 1.24) tocca inoltre un aspetto che il lettore vedrà emergere nella successiva vicenda del popolo: la condotta negativa delle guide del popolo (re e funzionari), anchessa causa della perdita della terra. Rispetto ai re d'Israele e Giuda, la cui condotta sarà aspramente criticata dagli autori della storia biblica e dai profeti, la condotta di Giosuè, fedele e obbediente successore del servo di Dio Mosè, configura il leader ideale del popolo.

7 Così L.D. Hawk, Joshua, pp. 4-5: «The narrator employs a number of devices to link Joshua to Moses and to signal a smooth succession of leadership. [...] The narrator also carefully separates the two men and implies Joshua's continuing subordination to Moses». Cf. anche S.B. Chapman, "Joshua Son of Nun: Presentation of a Prophet», in J.J. Ahn S.L. Cook (eds.), Thus Says the Lord, 13-26: «From the outset of the book of Joshua, the character of Joshua is predominantly styled as Moses redux» (1). 
Mentre, infatti, «l'autorità regale era onorata nel Vicino Oriente antico come un dono degli dei per accrescere la prosperità della comunità rafforzando la qualità delle relazioni tra sovrano e sudditi» e «il re, in qualità di recettore di questo dono dell'autorità, era l'agente terreno che esercitava la sovranità divina nella comunità per conto del re degli dei», ${ }^{8}$ per il Deuteronomio la regalità umana non è una manifestazione di quella divina, né la sua autorità è una sorta di delega conferita dal vero re. La signoria divina sul suo popolo si manifesta ora tramite un testo scritto (la Torah di Mosè), di cui si parla solo in Dt 17,14-20 entro la cornice del codice deuteronomico $(D t 12-26)^{9}$ e che rappresenta il lascito di Mosè, la cui funzione non è trasmissibile. Giosuè non è il nuovo Mosè (cf. Dt 34,10-12), mentre Dio continua a guidare il suo popolo e a esercitare la sua sovranità su di lui attraverso la Torah scritta (Gs 1,7-8). A questo scritto è ora assegnato il compito di guida e giudizio della storia, come lo stesso Mosè ammonisce (cf. Dt 28,58-61). ${ }^{10}$

Nel canone ebraico, i Profeti anteriori (Gs-2Re) sono introdotti con la figura di Giosuè, che basa il suo agire sul libro consegnato da Mosè (cf. Gs 1,8: sēper hattôrâ) e giungono alle battute conclusive con la scena del ritrovamento del libro della legge (sēper hattôrâ) sotto re Giosia in 2Re 22,8. Questo spiega perché, sulla scia di Nelson, Vignolo possa concludere che Giosuè è simultaneamente vera e propria pietra di paragone d'ogni futura figura regale e sapiente, che «spicca come un uomo-libro perfettamente paradigmatico e massimale, in quanto primo discepolo erede di Mosè e suo felice doppio originario, capace di compiere, prefigurare, e normare ogni altro uomo-libro - uomo-lettore - della storia salvifica»; in particolare «prefigura l'esemplare e insuperato re Giosia, a sua volta stilizzato come modello canonico». ${ }^{11}$ Come però osserva Levinson, ${ }^{12}$ la presentazione che la cosiddetta storia deuteronomistica fa del re Giosia contrasta nettamente con la normativa sul re di $D t 17,14-20$, la quale in pratica espone solo i limiti delle prerogative regali, escludendo in particolare il re dal culto e dall'amministrazione della giustizia:

The double denial by Deuteronomy of the conventional participation of the king in justice and cultus amounts to an extraordinary rejection of the standard Israelite

8 D. Launderville, Piety and Politics, p. 1.

9 Cf. S. Paganini, Deuteronomio. Nuova versione, p. 295.

10 Cf. F. Dalla Vecchia, «Il potere del re», pp. 79-98.

11 R. Vignolo, «Antitipo come controtipo. Giosuè "uomo-libro" pieno Salomone "uomo-libro" mancato», p. 195.

12 B.M. Levinson, «The Reconceptualization of Kingship in Deuteronomy, pp. 511-534 . 
and Near Eastern royal ideology. The Law of the King paradoxically denies him all the essential components of royal power and prestige: supreme judicial authority and sponsorship of the cult. Even military leadership is sharply curtailed. ${ }^{13}$

Proprio a questo livello emerge tuttavia un paradosso, poiché sebbene molti interpreti pensino che la riforma attuata dal re Giosia rappresenti il trionfo della legge deuteronomica,

«In fact, the actual story is more complex, for Deuteronomy's norms are implemented by the Deuteronomistic Historian in a completely non-Deuteronomic way. The double denial by the Deuteronomic author that there should be any connection between king and cult is reversed by the Deuteronomistic Historian». ${ }^{14}$

Levinson mostra infatti che la presentazione che i Libri dei Re fanno del re Giosia lo vede implicato in una riforma del culto; degna di nota è specialmente la celebrazione della Pasqua a Gerusalemme, celebrata per ordine del re $(2 R e 23,21-25)^{15}$. In particolare Levinson rimarca che in un certo senso i Libri dei Re ridimensionano la prospettiva del Deuteronomio, pur volendo implementare le norme in esso contenute. Su questa base, Levinson argomenta che il Deuteronomista di fatto finisce per abrogare le esigenze del Deuteronomio, fornendo nuovo impulso alla monarchia. ${ }^{16}$

Senza entrare nel dettaglio della proposta di Levinson, mi pare evidente che appunto la figura di Giosuè esposta nel libro omonimo, a differenza di Giosia, corrisponda perfettamente a quanto la legge sul re di $D t 17,14-20$ richiede al leader del popolo. Non è dunque una sorta di anticipazione di Giosia che

13 Ibidem, p. 524.

14 Ibidem, p. 525.

15 «The Deuteronomistic account of the national celebration of the Passover in Jerusalem, the high point of the account of Josiah's reform, radically revises the Deuteronomic Passover, which it ostensibly enacts to the letter (2 Kgs. xxiii 21-25). In narrating the first ever centralized celebration of the Passover, the Deuteronomistic Historian makes Josiah the royal patron of the cult who assiduously supervises the celebration of the Passover. $\mathrm{He}$ it is, then, standing ceremoniously before the assembled people, who solemnly com-

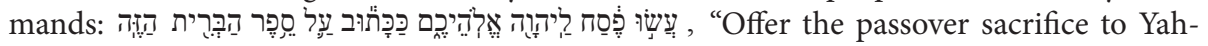
weh, your God, as it is written upon this scroll of the covenant" (2 Kgs. xxiii 21)» (ibidem», p. 525).

16 Ibidem, p. 534. 
spiega il protagonista, ${ }^{17}$ ma la prospettiva globale che sorregge il libro, la quale non va compresa come epopea di conquistatori, benché tale sia l'impressione di superficie. Come ha mostrato la storia della composizione del libro di Giosuè, ${ }^{18}$ non è possibile considerarlo contemporaneo agli avvenimenti, nel qual caso si tratterebbe di un monumento scritto per celebrare le imprese compiute dal grande eroe e dagli ebrei della sua generazione, non dissimile nelle sue motivazioni dalle numerose iscrizioni e cronache reali ritrovate negli scavi del Vicino Oriente. Diversamente, se invece, come proposto da molti, ${ }^{19}$ fosse stato scritto allepoca del re Giosia - il cui nome ebraico è tra l'altro affine a quello di Giosuè - avrebbe potuto fungere da manifesto della pretesa di quel re di riunire sotto un'unica guida politica l'intera popolazione che riconosceva come passato unitario la vicenda esodica e il riferimento al Dio nazionale YHWH. Infine, come pensa l'ipotesi della storiografia deuteronomistica, ${ }^{20} \mathrm{il}$ libro potrebbe essere destinato alla generazione dell'esilio per mostrare che la terra, donata da YHWH, fedele alle promesse fatte ai padri e al patto stipulato al Sinai, era stata conquistata in una campagna militare condotta dallo stesso YHWH durante la generazione di Giosuè che si era dimostrata fedele agli ordini divini; nello stesso tempo, la narrazione di Giosuè contiene i messaggi che il popolo in esilio (o sotto dominazione straniera) deve fare propri (Gs 23-24): il possesso della terra era condizionato all'osservanza delle norme del patto sinaitico, clausola che le generazioni successive non hanno saputo rispettare (come preannuncia Giosuè in 23,11-16;24,19-20), procurando così la disfatta totale e la perdita

17 Come, richiamandosi a Nelson, propone E.A. Knauf, Josua, p. 17: Giosuè è persona isolata a livello tribale e genealogico e questo porta a concludere che il suo nome sia in realtà una deliberata variante del nome Giosia.

18 Per una rassegna sulla storia della redazione, cf. T.C. Butler, Joshua 1-12, pp. 79-84, con una conclusione non del tutto condivisibile: «Ultimately, a historian in the time of the united monarchy created the individual book of Joshua as a call to unity between the forces of David and those of Saul and ultimately between David/Solomon and rebellious forces in the northern tribes. Slightly later, the editor of Judges incorporated parts of Joshua into his work and created a pro-Judean work aimed against the revolt of Jeroboam I. Still later, an editor joined Joshua/Judges into an ultimate history reaching through Kings» (84); il libro rivela inoltre ritocchi successivi (minimi) da parte di scribi.

19 Cf. recentemente E.A. Knauf, Josua, pp. 17-20, secondo il quale la più antica edizione di Giosuè risalirebbe al regno di Giosia o più tardi; il libro è letteratura tradizionale, opera di generazioni di scribi e di esperti nella Scrittura (Schriftgelehrten); molti intervennero con cambi minimi, su un singolo versetto o poco più; altri riformularono di nuovo la tradizione alla luce di un nuovo programma politico o teologico; il libro iniziò attorno al 600 a.C. a Betel o Gerusalemme; la redazione finale avvenne a Gerusalemme poco dopo il 400, tranne un ritocco anti-samaritano nel III o II secolo (Knauf isola almeno sette redazioni nel libro).

20 Cf. H.N. Rösel, Joshua, pp. 3-7. 
della terra. In quest'ultima prospettiva, Giosuè rappresenterebbe il leader che condurrà la nuova comunità che sorgerà dopo l'esperienza dell'esilio a rioccupare la terra: né capo religioso, né militare e neppure giudiziale, ${ }^{21}$ esattamente come il re in $D t 17$.

In tutte queste ipotesi risalta che non si tratta di una storia scritta dai vincitori, ma da un popolo che ha sperimentato il fallimento, in particolare nel confronto con i grandi imperi, la cui propaganda non di rado era in grado di minare dalle fondamenta le certezze suscitate dalla fede in Yhwh (come bene esemplifica il discorso del Gran Coppiere in 2Re 18,17-35). A scrivere sono gli oppressi o quelli espropriati della propria terra e costretti a vivere in esilio. Emerge inoltre qui una caratteristica della storiografia biblica, che la accomuna con quella greca; ${ }^{22}$ anche questa storiografia è infatti l'espressione letteraria della reazione di una cultura specifica alla propaganda degli imperi orientali, la quale era effettuata sia tramite iscrizioni monumentali sia tramite annali e cronache reali. ${ }^{23}$ La porzione dell'AT che meglio esprime la reazione agli imperi antichi è certamente costituita dalla letteratura profetica, ma lo stesso va affermato dei testi storici, nei quali emerge la consapevolezza d'Israele di svolgere entro il contesto politico mondiale un ruolo peculiare, fondato sul rapporto che si è stabilito lungo la storia tra questo popolo e YHWH. Il confronto con gli imperi è inoltre il motivo dominante della narrazione che costituisce il cuore della Torah, cioè il conflitto tra YHWH e l'Egitto; tale confronto troverà rinnovata riproposizione con l'apparire sullo scenario storico degli imperi neo-assiro, neo-babilonese e persiano.

Nella stessa prospettiva, anche le affermazioni del Deuteronomio sul re non vanno comprese come un'antica teoria politica preservata nella memoria del popolo, ${ }^{24}$ bensì come la deliberata presa di posizione di fronte a eventi storici che hanno fatto luce sulle cause della fine tragica dell'esperienza monarchica,

21 S.B. Chapman, "Joshua Son of Nun: Presentation of a Prophet», 13-26 pensa a un profeta, ma si tratta di una rilettura possibile della tradizione su Giosuè, selezionando alcuni passaggi; l'insieme del libro non conforta tale assunto; cf. l'illuminante sintesi sugli sviluppi letterari delle figura di Giosuè in A. Rofé, «Joshua Son of Nun in the History of Biblical Tradition», p. 333-364 (ebraico).

22 Si veda specialmente A. Momigliano, Le Radici classiche della storiografia moderna.

23 Sulla propaganda degli imperi vicino-orientali, cf. M. Liverani, Guerra e Diplomazia nell'Antico Oriente. Un esempio di questa reazione è efficacemente esposto in E. Otto, «Mosè e la legge. Il personaggio di Mosè», pp. 53-104.

24 Come propone N. Lohfink, «Separazione dei poteri», pp. 65-85; Lohfink parla di «progetto di costituzione» (p. 74) e di «teoria costituzionale» che «non ha mai trovato una realizzazione concreta» (p. 78); cf. R.D. Nelson, Deuteronomy. A Commentary, p. 210: «Constitutional proposals»; S. Paganini, Deuteronomio. Nuova versione, introduzione e com- 
bene evidenziate nelle riflessioni sulla caduta del regno d'Israele (ma applicate anche a Giuda) in 2Re 17,7-23; queste cause sono già enunciate in forma di maledizioni conclusive alla stipulazione del patto in Dt 28,15-69.

Tutto ciò illumina anche circa la prospettiva che sorregge il libro di Giosuè, nel quale risalta l'insistenza sulla presentazione d'Israele come un tutto compatto, sotto un'unica guida e fedele agli ordini impartiti da Dio. Tale visione costituisce un chiaro insegnamento, oltre che un monito: Yhwh realizza le sue promesse, donando una terra, che tuttavia si può perdere, se non si rimane a lui fedeli (cf. specialmente Gs 23-24). Giosuè rappresenta un modello di guida che non troverà più un vero e proprio seguito, implicitamente preparando il lettore della storia successiva all'esito nefasto della avventura monarchica; la legge data a Mosè sul Sinai è il punto di riferimento, costantemente richiamato, dei capi e del popolo: osservando la legge, Israele dimostra la sua fedeltà al patto con YHWH, ma il libro si chiude appunto con la previsione dell'infedeltà del popolo al Dio geloso, il quale, come ha realizzato le sue promesse, ora potrebbe adempiere anche le maledizioni accluse al patto se il partner umano si dimostrasse infedele (cf. Gs 23,6-16; 24,19-20). Il libro culmina, infatti, nell'invito rivolto al popolo a una sollecita riconoscenza nei confronti di Colui che gli ha donato il paese promesso agli antenati. Il narratore pone l'accento dunque su quanto Dio ha realizzato, così come rileva che ogni azione intrapresa da Giosuè rappresenta l'esecuzione fedele di ordini impartiti dal Dio che l'ha designato quale guida del suo popolo.

L'insistenza sulla fedele esecuzione degli ordini divini da parte di Giosuè e sulla corrispondente condotta del popolo - escluso Akan (Gs 7) - colloca il resoconto della conquista in una peculiare posizione: in effetti, né la generazione dell'esodo né le successive generazioni (cf. i libri da Giudici a Re) manifestano una tale propensione. In certo qual modo la vicenda narrata in Giosuè è parallela a quella di Abramo e presenta analogie interessanti: in entrambi i casi si tratta degli inizi nel paese. La fedeltà e l’obbedienza di Abramo culminano nell'ottenimento di una discendenza (anche se si tratta di un solo figlio), così la fedeltà e lobbedienza del popolo culminano nel dono della terra, il quale, come mostrano le pagine finali, sollecita il popolo a decisione libera e responsabile nei confronti di YHWH (Gs 24). È significativo, infatti, che il nostro libro, come il Deuteronomio, pur enfatizzando il dono della terra, sottolinei al contempo il rischio della perdita futura della stessa: «Badate dunque attentamente a voi stessi, amando Yhwh vostro Dio. Se, però, vi allontanerete e vi unirete al resto di queste nazioni rimaste con voi, imparentandovi con loro e assimilandovi

mento, p. 284: «Strutture statali in un sistema teocratico». Si veda la diversa valutazione in J.-M. Carrière, «Le pouvoir de tout un chacun (Dt 16,18-18,22)», pp. 43-61. 
reciprocamente, state certi che Yhwh non scaccerà più queste nazioni davanti a voi ed esse diverranno per voi una rete, una trappola, un flagello ai vostri fianchi e spine nei vostri occhi, finché non scomparirete da questa buona terra che Yhwh vostro Dio vi ha dato» (Gs 23,11-13). Chi scrive conosce già il dramma del fallimento, cosicché fa pronunciare al protagonista del libro un'esortazione che spiega tale fallimento: si tratta della punizione perché il popolo si è allontanato dal suo Dio e ha adorato altre divinità; in tal modo il libro diventa un appello e un programma per una 'nuova' vita nella terra: ${ }^{25}$ come la disobbedienza ha provocato la perdita della propria terra, così l'obbedienza consentirà nuovamente al popolo di rientrarne in possesso.

La figura del protagonista non si spiega dunque alla luce del rapporto con il suo predecessore, ma neppure alla luce delle attuazioni storiche che la storiografia deuteronomistica sembra suggerire, in particolare Giosia. La comunità che attende la futura attuazione delle promesse divine di rinnovamento non sarà di nuovo guidata da un re, ma avrà come riferimento permanente - per le guide e per i membri - il libro, e chiunque sarà chiamato ad assumere il ruolo di guida dovrà, come Giosuè, ascoltare, meditare e mettere in pratica la Torah.

\section{Bibliografia (solo quella citata nel contributo)}

Béré P., Le second Serviteur de Yhwh. Un portrait exégétique de Josué dans le livre éponyme (OBO 253), Vandenhoeck \& Ruprecht, Göttingen 2012.

Budd P.J., Numbers (WBC 5), Word Books, Waco (TX) 1984.

Butler T.C., Joshua 1-12 (WBC 7A), Zondervan, Grand Rapids (MI) ${ }^{2} 2014$.

Carrière J.-M., "Le pouvoir de tout un chacun (Dt 16,18-18,22)», in D. Luciani A. Wénin (edd.), Le Pouvoir. Enquêtes dans l'un et l'autre Testament (LD 248), Cerf, Paris 2012, 43-61.

Chapman S.B., "Joshua Son of Nun: Presentation of a Prophet», in J.J. Ahn - S.L. Cook (eds.), Thus Says the Lord. Essays on the Former and Latter Prophets in Honor of Robert R. Wilson (LHB/OTS 502), T \& T Clark, New York-London 2009, $13-26$.

Dalla Vecchia F., Giosuè. Introduzione, traduzione e commento (Nuovissima versione della Bibbia dai testi antichi 6), San Paolo, Cinisello Balsamo (MI) 2010.

Dalla Vecchia F., «Il potere del re», in G. Canobbio - F. Dalla Vecchia - R. Maiolini (edd.), Il potere (Quaderni Teologici del Seminario di Brescia 24), Morcelliana, Brescia 2014, 79-98.

Hawk L.D., Joshua (Berit Olam), Liturgical Press, Collegeville (MN) 2000.

Knauf E.A., Josua (ZBK), Theologischer Verlag, Zürich, 2008.

25 Cf. G. Hentschel, «Il libro di Giosuè», p. 365. 
Launderville D., Piety and Politics. The Dynamics of Royal Authority in Homeric Greece, Biblical Israel, and Old Babylonian Mesopotamia, Eerdmans, Grand Rapids (MI) 2003.

Levine B.A., Numbers 21-36. A New Translation with Introduction and Commentary (AncB 4A), Doubleday, New York (NY) 2000.

Levinson B.M., «The Reconceptualization of Kingship in Deuteronomy and the Deuteronomistic History's Transformation of Torah», VT 51 (2001) 511-534.

Liverani M., Guerra e Diplomazia nell'Antico Oriente, Laterza, Roma-Bari 1994.

Lohfink N., «Separazione dei poteri. Le leggi del Deuteronomio sugli uffici come progetto di una costituzione che prevede la separazione dei poteri e il diritto canonico cattolico», in Id., Le nostre grandi parole. LAAntico Testamento su temi di questi anni, Paideia, Brescia 1986, pp. 65-85.

Momigliano A., Le Radici classiche della storiografia moderna. Sather Classical Lectures, Sansoni, Firenze 1992.

Nelson R.D., «Josiah in the Book of Joshua», JBL 100 (1981), 531-540.

Nelson R.D., Joshua. A Commentary (OTL), Westminter John Knox Press, Louisville (KY) 1997.

Nelson R.D., Deuteronomy. A Commentary (OTL), Westminster John Knox Press, Louisville-London 2002.

Otto E., «Mosè e la legge. Il personaggio di Mosè come progetto di teologia politica antitetico all'ideologia reale neoassira del VII sec. a.C.», in Id., (ed.), Mosè. Egitto e Antico Testamento (StBib 152), Paideia, Brescia 2006, 53-104.

Paganini S., Deuteronomio. Nuova versione, introduzione e commento (I libri biblici. Primo Testamento 5), Paoline, Milano 2011.

Porter J., «The Succession of Joshua», in J. Durham - J. Porter (eds.), Proclamation and Presence. Old Testament Essays in Honour of Gwynne Henton Davies, SCM Press, London 1970, 102-132.

Rofé A., «Joshua Son of Nun in the History of Biblical Tradition», Tarbiz 73(2003/4), 333-364 (ebraico).

Rösel H.N., Joshua (HCOT), Peeters, Leuven 2011.

Schäfer-Lichtenberger Ch., Josua und Salomo. Eine Studie zu Autorität und Legitimität des Nachfolgers im Alten Testament (VT.S 58), Brill, Leiden 1995.

Vignolo R., "Antitipo come controtipo. Giosuè "uomo-libro" pieno Salomone "uomolibro" mancato», in G. Benzi - D. Scaiola - M. Bonarini (edd.), La profezia tra l'uno e l'altro Testamento. Studi in onore del prof. Pietro Bovati in occasione del suo settantacinquesimo compleanno (AnBib-Studia 4), G \& BP, Roma 2015, 191-203.

Zenger E. (ed.), Introduzione all'Antico Testamento Queriniana, Brescia ${ }^{3} 2013$. 